\title{
Experimental and Numerical Study of the effect of Gas-shrouded Plasma Spraying on Cathode Coating of Alkaline Electrolysis Cells
}

T. Liu and A. Ansar (German Aerospace Center), Düsseldorf / D

Dual layer electrode coating for alkaline water electrolysis was prepared by plasma spraying. For improving performance this work aims at reducing the oxide in electrode coating. Regarding the necessity of obtaining high specific area, atmospheric plasma spray was employed under protection of argon which was used as shrouding gas. Composite cathode was established on Ni-coated perforated steel sheet with crushed and gas atomized Nickel-based alloy powders. The dual-layer structure was a composite of 5 layers of NiAl at the bottom and 10 layers of NiAIMo as the top layer. Microstructure and morphology were studied by scanning electron microscope (SEM). Element content was estimated by energy dispersive X-ray spectrometer (EDS). Enthalpy probe was introduced for measuring plasma temperature and velocity as well as gas composition. Numerical calculation was carried out with same condition for better understanding the shrouding effect. The results showed moderate protection by using of arranged gas shrouding. Overall the dual layer region, Oxygen was decreased by $0.3 \%$ from $3.46 \%$ to $3.15 \%$. With gas shrouding coating exhibited similar element contents as coating sprayed by VPS. However, no obvious difference was observed in microstructure and morphology with or without gas shrouding.

1

\section{Introduction}

As a clean energy carrier, hydrogen has drawn a lot of interests in industry and in academic research.

Especially, the global warming effect brings challenge in environment and society in recent years. As one of the most promising approaches to produce hydrogen alkaline water electrolysis (AWE) exhibits surprising potential of taking advantage of the renewable energy as well as the capacity to be integrated in large-scale energy storage [1]-[4]. It has been intensively investigated to reduce the cost and decrease the degradation of the electrodes as low as possible throughout the development [1], [5]-[7]. The state-ofart cathode is based on nickel alloy which shows low over-potential and moderate current density for hydrogen evolution reaction (HER) [7]-[9]. Recently, a lot of works have been reported focusing on the process to prepare cathode with reasonable performance. Kjartansdóttir et al [3] tested a physical vapour deposited (PVD) Al/Ni electrode with overpotential of $-1000 \mathrm{mV}$ at $500 \mathrm{~mA} / \mathrm{cm} 2$. Manazoğlu et al [10] reported over-potential around $-200 \mathrm{mV}$ at current density of $100 \mathrm{~mA} / \mathrm{cm} 2$ on an electroplated $\mathrm{Ni} /$ Mo electrode. While Schiller et al [11], [12] presented much better performance with an overpotential as low as $-90 \mathrm{mV}$ at $500 \mathrm{~mA} / \mathrm{cm} 2$ current density based on VPS coated NiAIMo electrode.

Methodology and installations

\section{$2.1 \quad$ Experimental setups}

\subsubsection{Plasma spray installation}

This work was carried out in a chamber which was able to be controlled in vacuum state or in atmospheric state by a vacuum pump/ventilation pump (see Fig. 1). The pressure of the chamber could be controlled either as low as $6000 \mathrm{~Pa}$ for VPS or be maintained at $1 \mathrm{~atm}$. $\left(9.4 \times 10^{4} \mathrm{~Pa}\right)$ for APS. Inside the chamber, a water-cooled F4-VB torch was installed on an X-Y movement (see Fig. 1 (b)). The feedstock was perpendicularly fed to the plasma jet through an injector with $1.8 \mathrm{~mm}$ internal diameter being installed $3 \mathrm{~mm}$ upstream to the nozzle exit. A gas shrouding part with 12 arranged shrouding nozzles was mounted to the torch and to prevent any unfavourable entering path of the ambient gas as indicated by $\mathrm{P} 1$ in Fig. 2(a). The ground-face of the shrouding part was aligned with the exit of the torch that the ambient gas would be well entrained to the plasma jet through the path P2 in Fig. 2(a). The gas used for shrouding was argon with net pressure as high as $3.76 \times 10^{5} \mathrm{~Pa}$; the input argon was delivered to the shrouding nozzles by two entrances as indicated in Fig. 2(b) and (c). In Fig. 2(d) the simplified nozzle was presented to be a V-like notch with an expanding angle of $30^{\circ}$.

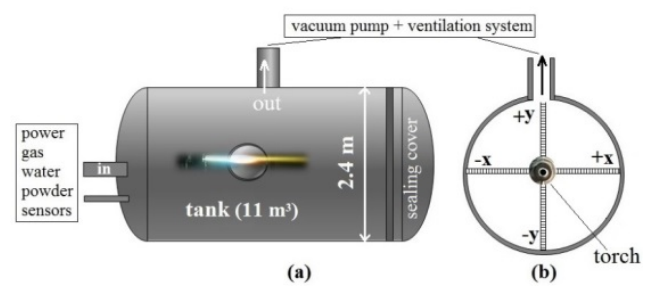

Fig. 1. the plasma spray installation with vacuum pump and ventilation pump

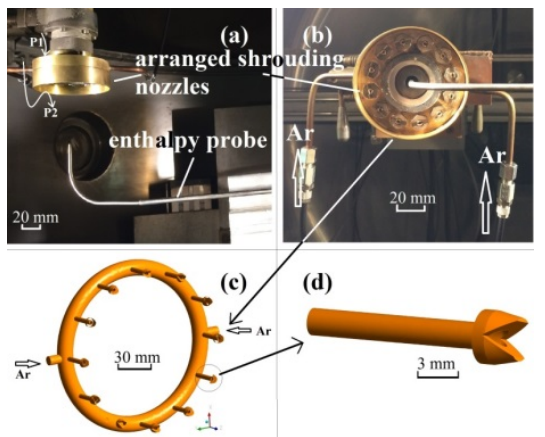

Fig. 2 installations of the arranged shrouding nozzles and the enthalpy probe: (a) top view; (b) front view; (c) model of the arranged nozzles with the gas delivering channel; (d) model of a single shrouding nozzle simplified for simulation

\subsubsection{Enthalpy probe}


A lot of works have been reported to successfully use enthalpy probe to diagnose plasma jet with reasonable precision [13]-[17].In this work the enthalpy probe system from TEKNA (Québec, Canada) was employed to detect the plasma gas composition, the plasma temperature and the plasma velocity. The probe used was ENT 476 with orifice area of $1.27 \mathrm{~mm}^{2}$ at the tip. The probe tip was placed on the centreline of the torch aperture and perpendicularly pointed to the centre of the torch. Instead of moving the probe the torch was precisely moved to adjust the measuring position for depicting the distribution of the gas composition as well as the temperature and the velocity of the plasma. The zero point was set as the centre of the plasma which was found by slowly moving the torch. During the measurement, the sampling rate was adjusted to achieve a good isokinetic of the sample gas for reducing error.

\subsubsection{Feedstocks}

The feedstock used in this work was commercially supplied $\mathrm{NiAl}(56 / 44 \mathrm{wt} \%)$ as the bond layer and NiAlMo (39/44/17 wt \%) as the top layer. The size distribution of the used powders was displayed in Fig. 3. The granularity test also indicated that the $d_{50}$ was $15.43 \mu \mathrm{m}$ and $16.67 \mu \mathrm{m}$ for NiAl and NiAIMo respectively. Both of the two powders were sphered to improve the flow-ability and thus to diminish the fluctuation in the powder feeding according to our prior work [18]. The morphology of the two powders was shown in Fig. 4.

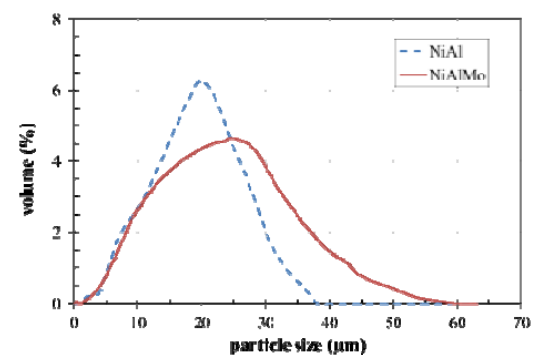

Fig. 3 the granularity of NiAl and NiAIMo

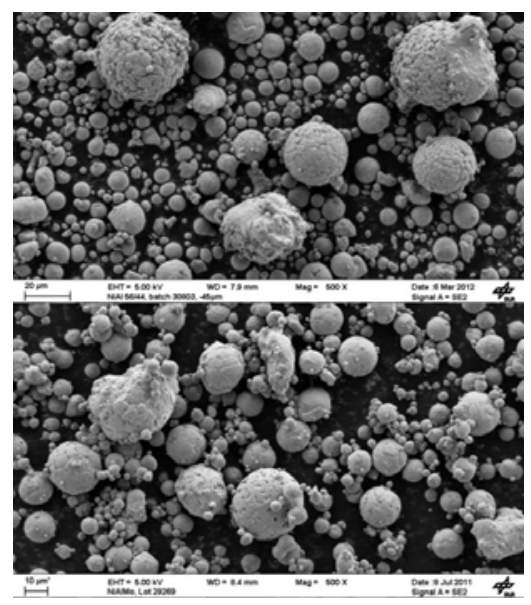

Fig. 4 the morphology of NiAl and NiAIMo

\subsubsection{Samples preparation and procedure}

Three samples were prepared with different pressure and different shrouding state (see Tab. 1). Sample 1 was sprayed in VPS $(6000 \mathrm{~Pa})$; sample 2 was obtained in APS (1 atm.) when the plasma jet was being shrouded by compressed argon with net pressure of $3.76 \times 10^{5} \mathrm{~Pa}$ (4 atm.); sample 3 was also prepared in APS but without plasma jet shrouding. The substrates used were stainless steel sheets with size of $\phi 32 \mathrm{~mm} \times 0.5 \mathrm{~mm}$ on which a pure nickel layer was pre-coated with thickness about $36 \pm 6 \mu \mathrm{m}$. The substrates were punched to have open pores with diameter of $2 \mathrm{~mm}$ which resulted in an effective area of $555 \mathrm{~mm}^{2}$ as indicated in Fig. 5. After being sandblasted, for each sample, 5 layers of NiAl were firstly sprayed and then 10 layers of NiAlMo were continuously deposited on the surface.

Tab. 1 operating parameters and shrouding state

\begin{tabular}{|c|c|c|c|c|c|c|c|}
\hline i & $\Xi$ & $\frac{\widehat{\sum}}{\frac{1}{\omega}}$ & $\begin{array}{l}\stackrel{\sum_{j}}{\omega} \\
\frac{0}{I}\end{array}$ & $\begin{array}{l}\widehat{\sum} \\
\stackrel{\vec{\omega}}{N} \\
\stackrel{N}{I}\end{array}$ & 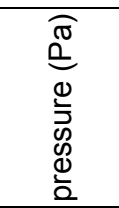 & 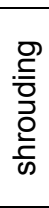 & 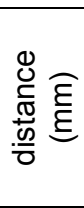 \\
\hline 1 & 420 & 45 & 10 & 4 & $6 \times 10^{3}$ & 0 & 300 \\
\hline 2 & 420 & 45 & 10 & 4 & $9.4 \times 10^{5}$ & 1 & 80 \\
\hline 3 & 420 & 45 & 10 & 4 & $9.4 \times 10^{5}$ & 0 & 80 \\
\hline
\end{tabular}

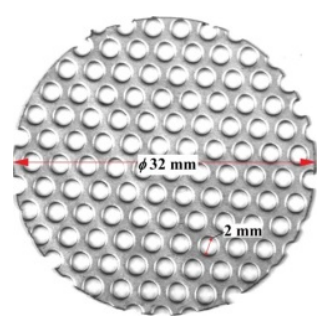

Fig. 5 the substrate used in this work

\subsection{Mathematical model}

\subsubsection{Simulation assumption and governing equations}

For verifying the effect of the gas shrouding on the plasma jet, numerical simulations were carried out focusing on the plasma jet behaviour as well as the ambient gas. Thus, the reaction between electrons and ions, such as charge transfer, was not mentioned. The magnetic force on particles and the current density on electrodes were also not interesting for this work. The simulation work was based on the following assumption: the plasma is in equilibrium; the profile of temperature and velocity of the plasma jet is in the form of Gaussian distribution; the plasma gas is a mixture of $\mathrm{Ar}$ and $\mathrm{H}_{2}$; all solid parts are rigid; the heat absorbing effect of the solid part was not considered; the entrained air was a mixture of $79 \% \mathrm{~N}_{2}$ and $21 \%$ $\mathrm{O}_{2}$; the properties of the ambient gas was constant 
with value determined according to the instant temperature.

Two governing equations were used [19]:

The conservation of mass:

$F \cdot(\rho U)=0$

The conversation of momentum:

$\nabla \cdot(\varphi v U)+\nabla p-\nabla \tau=0$

The conservation of energy:

$\nabla \cdot(\rho U h)-\sigma \nabla^{2} T=Q$

Where $\rho$ denotes the density, $\mathrm{kg} / \mathrm{m}^{3} ; \boldsymbol{U}$ gives the vector of velocity, $\mathrm{m} / \mathrm{s} ; p$ means the pressure, $\mathrm{Pa} ; \tau$ indicates the stress tensor, $\mathrm{Pa} ; T$ presents the temperature, $\mathrm{K}$; $h$ gives the enthalpy, $\mathrm{J} / \mathrm{kg}$; $\alpha$ is the thermal conductivity, $\mathrm{W} / \mathrm{m}^{2} \mathrm{k}$.

For closing the equations, the stress tensor $\tau$ in equation 2 is given as follow:

$$
\vec{\tau}=\mu\left[\left(\nabla U+\nabla U^{2}\right)-\frac{2}{\nabla} \nabla \cdot U I\right]-p I
$$

Where $\mu$ gives the viscosity, Pa.s; and I presents the unit tensor calculated as product of $A_{i k} A_{j k}$ which are two matrices automatically produced during the iteration [20].

Standard $k-\varepsilon$ model was employed for calculating the turbulence kinetic energy $k$ and the rate of the dissipation $\varepsilon$ according to the following equations [21]:

$$
\nabla \cdot(\rho k U)=\nabla \cdot\left[\left(\mu+\frac{\mu_{r}}{\sigma_{k}}\right) \nabla k\right]+G_{k:}+G_{k}-\rho-Y_{M}
$$

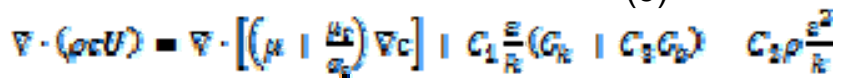

Where $G_{k}$ presents the generation of the turbulence kinetic energy from the mean velocity gradients; $G_{b}$ presents the turbulence kinetic energy generated by buoyancy; $Y_{m}$ gives the dedication of the fluctuating dilatation in the compressible turbulence to the dissipation rate; $C_{1}, C_{2}$ and $C_{3}$ are constants which are also able to be defined by user; $\mu_{t}$ denotes the turbulent viscosity which can be calculated as product of $0.09 \rho k^{2} / \varepsilon ; \delta_{k}$ and $\delta_{e}$ are the turbulent Prandtl numbers for $k$ and $\varepsilon$ with default value of 1 and 1.3 respectively [20].

\subsubsection{Computational domain and conditions}

The simulation in this work was focused on the behaviour of plasma jet and the effect of the gas shrouding. Thus the plasma was simplified as hot gas with given temperature and velocity entering the computational domain through the plasma inlet as indicated in Fig. 6. The nozzle for plasma gas inlet was created on the base of the F4-VB torch with adiabatic wall condition. The computational domain was mainly composed of the plasma inlet, the shrouding system and a cylinder with size of $\phi 120$ $\mathrm{mm} \times 200 \mathrm{~mm}$ for the progress of the off gas. For evaluating the entrainment of ambient gas to the plasma jet, gas composition at boundaries was defined as the volume fraction of corresponding component. That the plasma inlet was defined with $100 \%$ plasma gas and $0 \%$ air; the shrouding gas inlet was defined with $100 \%$ argon; the opening was defined with $90 \%$ air and $10 \%$ plasma gas; and the outlet was defined with $50 \%$ air and $50 \%$ plasma gas The temperature of the ambient gas at the opening and the outlet was defined to be $320 \mathrm{~K}$; while the shrouding gas was set at $300 \mathrm{~K}$. The distributions of the inlet temperature for the plasma gas were given as function of the coordinate $x$ and $y$ as follows:

$$
T(x, y)=15000 \times e^{-2: 98 \times 10^{2}\left(x^{2}+y^{2}\right)}
$$

However, the plasma inlet was defined as a mass inlet with flowrate of $0.00122183 \mathrm{~kg} / \mathrm{s}$ which was calculated according to the total flow rate of plasma gas and its density. Moreover, the plasma gas was simplified as a mixture of $90 \%$ argon and $10 \%$ hydrogen with a total flow rate of $50 \mathrm{NL} / \mathrm{min}$. Therefore, the plasma gas properties were considered as $90 \%$ from argon and $10 \%$ from hydrogen.

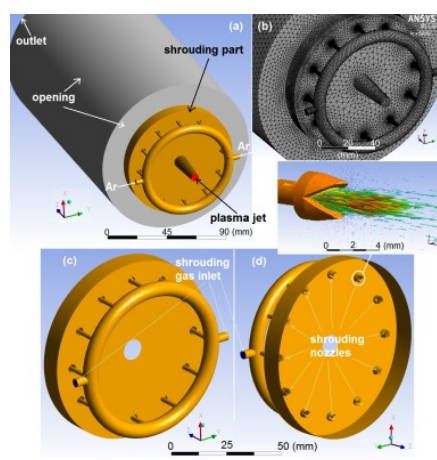

Fig. 6 model of simulation: (a) the mesh; (b) the model with 12-arranged nozzles for shrouding; (c) illustration of the result display and (d) a single nozzle for shrouding

\section{$3 \quad$ Results and discussions}

\subsection{Effect of shrouding gas on plasma jet}

The plasma jet was measured by the enthalpy probe at the centre which was determined by adjusting the torch to find the maximal value of the temperature and the velocity and the density (TVD). Then it was able to obtain plasma TVD with different standing on the centreline by moving back the probe following the spraying direction $(Z)$. In addition, in simulation case, the plasma TVD was able to be directly red out on the centreline. Consequently, the plots of the measured results and the simulated results were presented in Fig. 7 with lines presenting the simulation results (T_sim_SH0, T_sim_SH4...) and with discrete markers indicating the normal experimental results (T_exp_SH0, T_exp_SH4...). In the legend, "SHO" signified that no shrouding gas was used and "SH4" presented the use of shrouding gas with pressure of $4 \times 10^{5} \mathrm{~Pa}$. Accordingly, two aspects were clearly found that firstly the gas shrouding made the plasma jet different and secondly the simulation fit the experiment well. That the shrouding gas brought cooling effect and started to slightly reduce the plasma temperature at $70 \mathrm{~mm}$ on the spraying direction; while the plasma density was found to become higher. However, it is hard to point out how the plasma velocity had been changed, except that 
with shrouding gas the plasma velocity was found to be more instable. Similarly, the simulation also revealed the cooling effect of the shrouding gas on the plasma jet; while the velocities were found to be more or less the same.

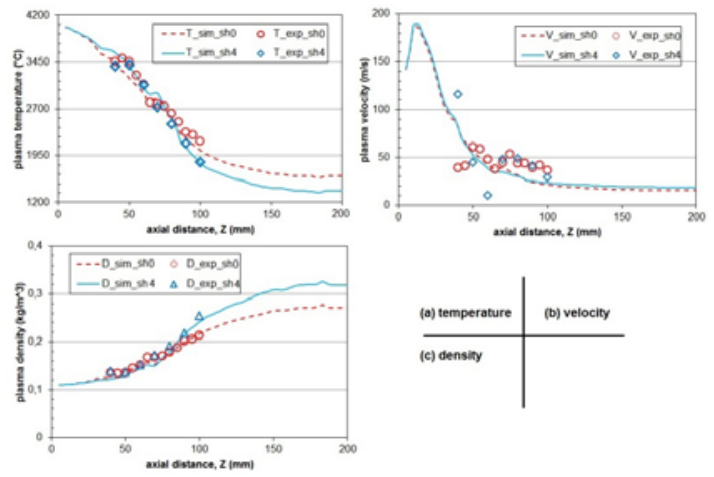

Fig. 7 measured and simulated characteristics of the plasma jet: temperature (a), velocity (b) and density (c)

The gas shrouding brought significate effect to the plasma gas composition as well as the surrounding gas. As shown in Fig. 8, the axial and radial distributions of $\mathrm{O}_{2}-\mathrm{N}_{2}$-Ar without gas shrouding were depicted with dashed lines and void markers; the distributions with gas shrouding were plot with solid lines and filled markers. It can be found that with gas shrouding the axial region between $70 \mathrm{~mm}$ and 100 $\mathrm{mm}$ was well protected to have higher argon fraction and lower oxygen fraction (see Fig. 8 (a)). That at the spraying distance $(80 \mathrm{~mm})$ the oxygen fraction was decreased from $3.2 \%$ to $0.9 \%$; while the argon fraction was increased from $96.8 \%$ to $99.1 \%$; and no $\mathrm{N}_{2}$ was detected. Radially, the argon-rich region around the plasma jet was obviously expended by the shrouding gas with edge being extended from $Y=-4$ $\mathrm{mm}$ to $Y=-10 \mathrm{~mm}$ (see Fig. 8 (b)). Without gas shrouding, the distributions of the volume fraction for argon and nitrogen were found to have a crossover at about $Y=-7 \mathrm{~mm}$; while with gas shrouding, the crossover was predicted at about $Y=-13 \mathrm{~mm}$.
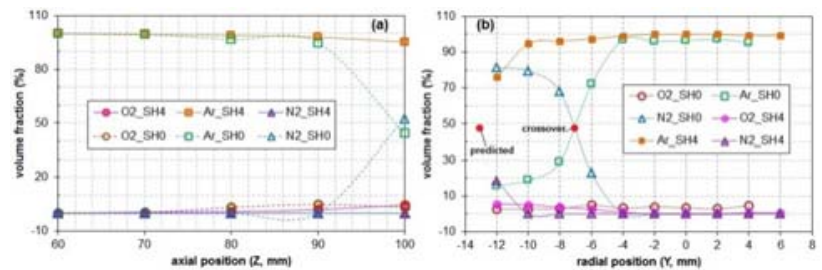

Fig. 8 measured gas composition along axial position $(Y=0 \mathrm{~mm})(\mathrm{a})$ and against the radial displacement $(Z=80 \mathrm{~mm})(\mathrm{b})$

Moreover, the shrouding effect was also confirmed by the simulation. As displayed in Fig. 9 (a) and (b), with gas shrouding the gas distributions were evidently modified. That in axial direction (see Fig. 9 (a)), the region between $50 \mathrm{~mm}$ and $170 \mathrm{~mm}$ of the centreline was protected with more argon while with less air $\left(\mathrm{O}_{2}\right.$ and $\mathrm{N}_{2}$ ). At $Z=80 \mathrm{~mm}$, the calculated volume fraction of oxygen reduced from $7.5 \%$ to about $2.4 \%$; and nitrogen was restricted with volume fraction as low as $9 \%$ which was only one third than that without gas shrouding; while the fraction of argon was increased from $57 \%$ to $83 \%$. It is clear from Fig. 9 (a) that without gas shrouding $\mathrm{Ar}$ _SHO intersected $\mathrm{N}_{2} \mathrm{SHO}$ at about $100 \mathrm{~mm}$ on the centreline while with gas shrouding the crossover was pushed away from the torch exit at about $150 \mathrm{~mm}$. In parallel, the simulation also gave reasonable results about the radial distribution of gas that the shrouding gas was able to optimize the spraying process by shielding the plasma jet from the air. However, due to the simplification of the calculating models, gap might exist between the simulation and the experiment. At least it gave possibility to justify the effect of the gas shrouding on the plasma jet.
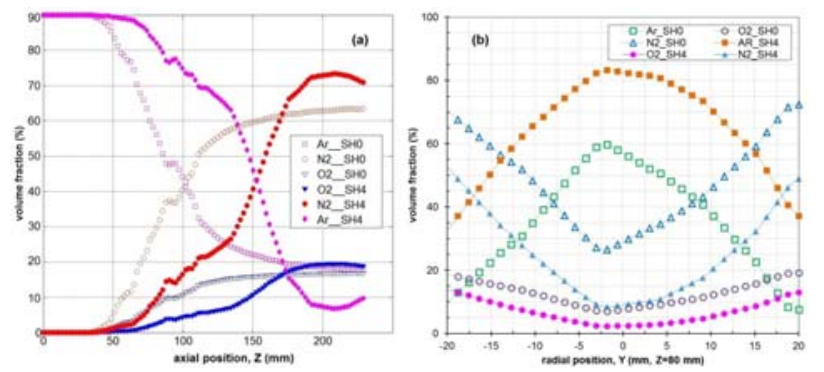

Fig. 9 the axial and radial distribution of $\mathrm{Ar}_{-} \mathrm{O}_{2} \mathrm{~N}_{2}$

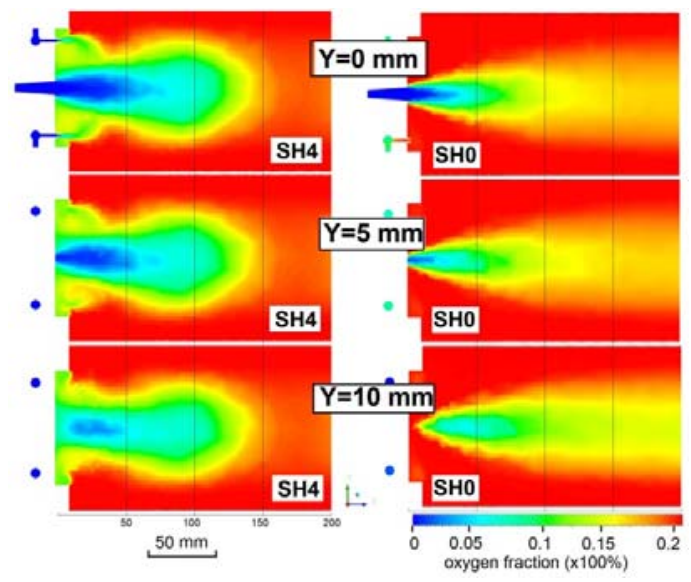

Fig. 10 the oxygen distribution on the selected planes

Additionally, simulation provided visible evidence about the shrouding effect. As shown in Fig. 10, the oxygen distributions on three planes, which were defined as $Y=0 \mathrm{~mm}, Y=5 \mathrm{~mm}$ and $Y=10 \mathrm{~mm}$, indicated that with gas shrouding the plasma jet was found to become radially wider and axially shorter with more region being protected from oxygen. Moreover, as depicted in Fig. 10, the shrouding gas released by the shrouding nozzles was simultaneously drawn toward the plasma jet due to the low pressure around the plasma jet. Therefore, a gas shield was formed around the plasma jet, and yet it is impossible to completely eliminate the entrainment of cold ambient gas due to the interval between the arranged nozzles 
(see Fig. 6). The shrouding part with arranged nozzles was designed as a compromise between the shrouding effect and the cooling effect. The stronger the shrouding effect is, the lower the temperature becomes. For example, with annular shrouding part, more shrouding gas will be required to obtain comparable gas shield as that of the arranged nozzles; moreover, the requirement of the shrouding pressure is necessary to be much higher to retain enough momentum of the shrouding gas for withstanding the entrainment of the ambient gas. Consequently, the plasma jet is well protected by the strong gas shield while the plasma temperature will be unavoidably reduced which is detrimental to the coating property. Therefore, the module with 12arranged-nozzles was finally employed in this work and it showed expected protecting effect to the plasma jet.

\subsection{Effect of shrouding gas on coatings characteristics}

For estimating the effect of gas shrouding on coating characteristics, microstructure and element composition of the coatings have been analysed by means of SEM and EDS. As mentioned above, the substrate was pre-treated to have a pure nickel layer for protecting the substrate and afterwards, NiAl coating was directly sprayed as a transition between the pure nickel layer and the NiAIMo layer according to the mass percent of nickel. Generally, the electrode of AWE will be activated to have high specific area by etching out Al before being put in service. That means the fraction of etchable $\mathrm{Al}$ in the as-sprayed coatings gives significant influence on the electrode performance. Therefore, by applying different spraying conditions, the performance of the resultant coatings will be different. As displayed in Fig. 11, sample 1, which was sprayed in vacuum, presented a structure obviously denser and thicker than sample 2 and sample 3 . While sample 2 and sample 3 exhibited similar structures with comparable pores being deeply trapped in NiAl layer. It is general accepted that big pores in the electrode coatings may bring unfavourable defect after long term working but its importance to the electrode performance is indistinct comparing with that of the nanostructure produced by etching. Therefore, the electrode performance will be mainly determined by the element composition of the coatings.

As a result of the gas shrouding, sample 2 presented a moderate mass percent of oxygen with a reduction of $0.31 \%$ comparing to the value of $3.46 \%$ in sample 3 (see Tab. 2). While, the content of Al in sample 3 was found to be lower than that of sample 2 . That meat after activation sample 2 might have more Nano pores than sample 3 and thus lead to a higher performance to hydrogen evolution. In addition, the content of $\mathrm{Ni}$ in sample 3 was found to be as high as $51.56 \%$ which was slightly higher than that of sample 1 and sample 2. However, in all cases the content of Mo was almost the same that meant during the spraying Mo was not oxidized. Moreover, regarding the content of $\mathrm{Al}$ in the two feedstocks (NiAl 56/44 and NiAIMo 39/44/17), it was able to estimate that the global content of $\mathrm{Al}$ in the as-sprayed coatings was about $44 \%$. And thus it was able to evaluate the loss of Al during the spraying for each sample, $5.38 \%$ in sample, $6.7 \%$ in sample 2 and $8.9 \%$ in sample 3 . The cause of the loss was mainly due to the vaporization of the in-flight particles and the deposition efficiency. Owing to the relative low boiling point, $\mathrm{Al}$ or Al-rich phase was easier to vaporize than $\mathrm{Ni}$ and Mo. Therefore, with higher oxide content and lower $\mathrm{Al}$, sample 3 was supposed to have the lowest electrode performance. Executing gas shrouding brought slight reduction of oxide in the coating and it was helpful for retaining Al.

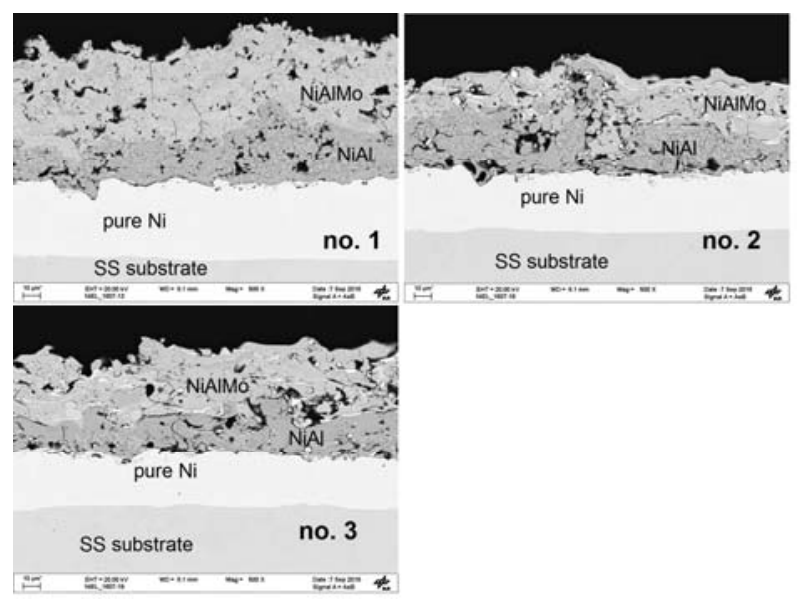

Fig. 11 microstructure of coatings in different cases

Tab. 2 mass percent of elements in different cases

\begin{tabular}{|l|l|l|l|l|}
\hline \multirow{2}{*}{ No. } & \multicolumn{4}{|l|}{ Mass percent (\%) } \\
\cline { 2 - 5 } & $\mathrm{O}$ & $\mathrm{Al}$ & $\mathrm{Ni}$ & $\mathrm{Mo}$ \\
\hline 1 & 1.8 & 38.62 & 48.94 & 10.64 \\
\hline 2 & 3.15 & 37.30 & 48.83 & 10.72 \\
\hline 3 & 3.46 & 35.11 & 51.56 & 9.87 \\
\hline
\end{tabular}

\section{$4 \quad$ Conclusions}

As a conclusion, in this work gas shrouding was carried out through a shrouding part with 12 arranged nozzles to protect the plasma jet. Both results of experiment and simulation showed that the shrouding gas was able to restrain the entrainment of oxygen to the plasma jet and retrain the oxygen fraction as low as $2.4 \%$ on the centreline at the spraying distance of $80 \mathrm{~mm}$. The plasma jet was surrounded by the shrouding gas and forced to become radially wider and axially shorter which were experimentally detected and successfully numerically predicted. Significant effects of gas shrouding on coating characteristics have been watched to retain $\mathrm{Al}$ in the coatings while reducing the oxide. The use of gas shrouding should be optimized to achieve gas shield with enough momentum while without bringing too much reduction to the plasma temperature. 


\section{References}

[1] Chen, L., Dong, X., Wang, Y., and Xia, Y. Separating hydrogen and oxygen evolution in alkaline water electrolysis using nickel hydroxide. Nature Communications 7, (2016), 11741-48.

[2] S. Marini et al., "Advanced alkaline water electrolysis," Electrochimica Acta, vol. 82, pp. 384391, Nov. 2012.

[3] C. K. Kjartansdóttir, L. P. Nielsen, and P. Møller, "Development of durable and efficient electrodes for large-scale alkaline water electrolysis," Int. J. Hydrog. Energy, vol. 38, no. 20, pp. 8221-8231, Jul. 2013.

[4] R. L. LeRoy, "Industrial water electrolysis: Present and future," Int. J. Hydrog. Energy, vol. 8, no. 6, pp. 401-417, 1983.

[5] M. Wang, Z. Wang, X. Gong, and Z. Guo, "The intensification technologies to water electrolysis for hydrogen production - A review," Renew. Sustain. Energy Rev., vol. 29, pp. 573-588, Jan. 2014.

[6] D. Pletcher and X. Li, "Prospects for alkaline zero gap water electrolysers for hydrogen production," Int. J. Hydrog. Energy, vol. 36, no. 23, pp. 1508915104, Nov. 2011.

[7] X. Tang, L. Xiao, C. Yang, J. Lu, and L. Zhuang, "Noble fabrication of $\mathrm{Ni}-\mathrm{Mo}$ cathode for alkaline water electrolysis and alkaline polymer electrolyte water electrolysis," Int. J. Hydrog. Energy, vol. 39, no. 7, pp. 3055-3060, Feb. 2014.

[8] T. Rauscher, C. I. Müller, A. Schmidt, B. Kieback, and L. Röntzsch, "Ni-Mo-B alloys as cathode material for alkaline water electrolysis," Int. J. Hydrog. Energy, vol. 41, no. 4, pp. 2165-2176, Jan. 2016.

[9] S. H. Zhou, Y. Wang, L.-Q. Chen, Z.-K. Liu, and R. E. Napolitano, "Solution-based thermodynamic modeling of the Ni-Al-Mo system using first-principles calculations," Calphad, vol. 46, pp. 124-133, Sep. 2014.

[10] M. Manazoğlu, G. Hapçı, and G. Orhan, "Electrochemical Deposition and Characterization of Ni-Mo Alloys as Cathode for Alkaline Water Electrolysis," J. Mater. Eng. Perform., vol. 25, no. 1, pp. 130-137, 2016.

[11] G. Schiller and V. Borck, "Vacuum plasma sprayed electrodes for advanced alkaline water electrolysis," Int. J. Hydrog. Energy, vol. 17, no. 4, pp. 261-273, Apr. 1992.

[12] G. Schiller, R. Henne, and V. Borck, "Vacuum plasma spraying of high-performance electrodes for alkaline water electrolysis," J. Therm. Spray Technol., vol. 4, no. 2, pp. 185-194, 1995.

[13] C. H. Chang and J. D. Ramshaw, "Computational study of high-speed plasma flow impinging on an enthalpy probe," Plasma Chem. Plasma Process., vol. 16, no. 1, pp. 17-38, 1996.

[14] J. R. Fincke, C. H. Chang, W. D. Swank, and D. C. Haggard, "Entrainment and demixing in subsonic thermal plasma jets: Comparison of measurements and predictions," Int. J. Heat Mass Transf., vol. 37, no. 11, pp. 1673-1682, Jul. 1994.
[15] M. Brossa and E. Pfender, "Probe measurements in thermal plasma jets," Plasma Chem. Plasma Process., vol. 8, no. 1, pp. 75-90, 1988.

[16] A. Capetti and E. Pfender, "Probe measurements in argon plasma jets operated in ambient argon," Plasma Chem. Plasma Process., vol. 9, no. 2, pp. 329-341, 1989.

[17] M. Rahmane, G. Soucy, and M. Boulos, "Analysis of the enthalpy probe technique for thermal plasma diagnostics," Rev. Sci. Instrum., vol. 66, no. 6, pp. 3424-3431, Jun. 1995.

[18] T. Liu and J. Arnold, "Study of in-flight particle stream and particle behavior for understanding the instability phenomenon in plasma spraying process," Surf. Coat. Technol., vol. 286, pp. 80-94, Jan. 2016.

[19] G. K. Batchelor, An Introduction to Fluid Dynamics. Cambridge University Press, 2000.

[20] N. Phan-Thien, "Tensor Notation," in Understanding Viscoelasticity: An Introduction to Rheology, N. Phan-Thien, Ed. Berlin, Heidelberg: Springer Berlin Heidelberg, 2013, pp. 1-27.

[21] B. E. Launder and D. B. Spalding, Lectures in mathematical models of turbulence [by] B. E. Launder and D. B. Spalding. London, New York: Academic Press, 1972. 